\title{
Transmit Beamforming with Cooperative Base Stations
}

\author{
Boon Loong Ng, Jamie S. Evans, Stephen V. Hanly \\ ARC Special Research Centre for Ultra-Broadband \\ Information Networks (CUBIN) \\ Department of Electrical and Electronic Engineering \\ University of Melbourne, VIC 3010, Australia \\ Email: \{b.ng, jse, hanly\}@ee.unimelb.edu.au
}

\author{
Defne Aktas \\ Department of Electrical and Electronics Engineering \\ Bilkent University, Bilkent, Ankara 06800, Turkey \\ Email: daktas@ee.bilkent.edu.tr
}

\begin{abstract}
We consider a cellular network where base stations can cooperate to determine the signals to be transmitted on the downlink. In such a scenario, it would be possible to use "macroscopic" transmit beamforming to improve system performance. The downlink beamformer of interest is generalised from some transmit beamformers that have been shown to meet various optimality criteria in the literature. The particular downlink beamformer structure enables us to recast our downlink beamforming problem as a virtual LMMSE estimation problem. Based on this virtual set up, we exploit the structure of the channel and develop distributed beamforming algorithms using local message passing between neighbouring base stations. Two algorithms are outlined, both of which are based on the Kalman smoothing framework. The first algorithm is a forward-backward algorithm that produces optimal performance, but it has the disadvantage of a delay that grows linearly with array size. The second algorithm, which is a limited extent algorithm, solves the delay problem by using only local information.
\end{abstract}

\section{INTRODUCTION}

The capacity of a cellular network is often limited by cochannel interference. In the uplink, sophisticated multiuser receivers can be implemented at the base stations (BS) to counter the co-channel interference. However, on the downlink, the receivers at the mobile stations (MS) are usually required to be simple, therefore complex interference suppression/cancellation techniques are not always possible at the receiver side. In this case, preprocessing at the transmitter side, such as downlink beamforming, can be an important means to improve system performance. In this paper, we are interested in a cellular network where BSs can cooperate to determine the signals to be transmitted on the downlink, effectively realising a "macroscopic" transmit beamforming scheme.

We consider a cellular network where MSs within a cell do not interfere with each other, either through time or frequency division multiplexing. However, we allow intercell interference by deploying full time or frequency reuse in every cell. The intercell interference is assumed to be highly localised, i.e. interference only comes from direct neighbours. There are dedicated communication links, free of interference, between neighbouring $\mathrm{BSs}$, thus enabling a cooperative sharing of information between such BSs. We focus on the downlink operation, whereby each BS in the network wishes to transmit data symbols to its active MS. Our aim in this paper is to design an efficient distributed downlink beamforming algorithm using local message passing between BSs, to overcome the impact of intercell interference.

Downlink beamforming for both single cell and multicell environments has been extensively researched in recent years [1]. A wide variety of optimality criteria have been explored, such as the minimisation of the mean square error (MSE) [2][7] and the maximisation of the worst signal-to-interferenceand-noise ratio (SINR) [8]-[10]. Another common optimality criterion is the power minimisation, which is often coupled with the minimum SINR constraints [8], [9], [11]-[13]. Many transmit beamformers derived from these various optimality criteria share a common structure, which resembles the linear minimum mean square error (LMMSE) estimator for a virtual model. The same structure also arises in [14], based on regularisation of the zero-forcing transmit beamformer. One of the main contributions in this paper is to recognise that this fairly widespread transmit beamformer can be recast as the solution to a simple LMMSE estimation problem for a virtual model. This recasting of the problem enables us to develop distributed transmit beamforming algorithms following the techniques presented in [15].

In this paper, our transmit antenna array is formed from antennas at multiple base stations. Downlink beamforming utilising multiple BSs has been considered in [11], [13], [16][18], however the proposed solutions usually assume that there is a central processing centre or controller to process information or coordinate information exchange among BSs. Our main contribution in this paper is to demonstrate ways to implement the downlink beamformer in a truly distributed manner through message passing between neighbouring BSs, by exploiting the local interference structure of the channel.

For simplicity, we shall restrict our attention to a onedimensional cellular network throughout this paper, which we call the linear array model. The assumption of local interference means that our channel possesses a Markov structure. As a result, a state space model based on the virtual LMMSE estimation problem can be formulated. Based on the state space model, we propose a distributed beamforming algorithm adapted from the Kalman smoothing framework, called 
the forward-backward beamforming algorithm. The forwardbackward algorithm produces the optimal transmitted signals for each BS, however the algorithm suffers from a delay that grows linearly with the network size. In order to overcome the delay problem, a limited extent distributed beamforming algorithm that is able to achieve near optimal performance with substantially reduced delay is proposed.

The rest of the paper is organised as follows. In Section II, our linear array model as well as a common transmit beamformer structure which meets various optimality criteria are presented. In Section III, we show how the beamformer structure allows us to recast the downlink beamforming problem as a LMMSE estimation problem. Our distributed transmit beamforming algorithms are presented in Section IV, together with some simulation results on the performance. Finally, we conclude our contribution in Section V.

Note that we use boldface lower and upper case letters to denote vectors and matrices respectively, and $\boldsymbol{I}$ denotes the identity matrix. The $i$ th element of the vector $\boldsymbol{a}$ is $a_{i}$ and we use $\boldsymbol{A}^{T}$ and $\boldsymbol{A}^{-1}$ to denote the transpose and the inverse of matrix $\boldsymbol{A}$ respectively.

\section{System Model AND Downlink BEAmForming}

We consider a one-dimensional cellular model (Fig. 1), called the linear array model, which can be expressed as

$$
y_{n}=\alpha_{n-1,+} x_{n-1}+\alpha_{n} x_{n}+\alpha_{n+1,-} x_{n+1}+w_{n}
$$

for $n=1, \cdots, N$ where $y_{n}$ is the received signal at the MS in cell $n, x_{n}$ is the transmitted signal from the $\mathrm{BS}$ in cell $n$ and we assume $x_{n}$ is zero for $n<1$ and $n>N$ ( $N$ is the array size). The $\left\{w_{n}\right\}$ are i.i.d. Gaussian random variables with zero mean and variance $\sigma^{2}$. The path gain from $\operatorname{BS} n$ to MS $n$ is $\alpha_{n}$, whereas $\alpha_{n,+}$ and $\alpha_{n,-}$ represent the severity of intercell interference from BS $n$ to MS $n+1$ and from BS $n$ to MS $n-1$ respectively. An equivalent representation is the following vector form:

$$
\boldsymbol{y}=\boldsymbol{H} \boldsymbol{x}+\boldsymbol{w}
$$

where

$$
\begin{aligned}
\boldsymbol{y} & =\left[\begin{array}{llll}
y_{1} & y_{2} & \cdots & y_{N}
\end{array}\right]^{T} \\
\boldsymbol{x} & =\left[\begin{array}{llll}
x_{1} & x_{2} & \cdots & x_{N}
\end{array}\right]^{T} \\
\boldsymbol{w} & =\left[\begin{array}{llll}
w_{1} & w_{2} & \cdots & w_{N}
\end{array}\right]^{T} .
\end{aligned}
$$

The channel matrix, $\boldsymbol{H} \in \mathbb{R}^{N \times N}$, is a tridiagonal matrix, given by

$$
\boldsymbol{H}=\left[\begin{array}{ccccccc}
\alpha_{1} & \alpha_{2,-} & 0 & 0 & 0 & \ldots & 0 \\
\alpha_{1,+} & \alpha_{2} & \alpha_{3,-} & 0 & 0 & \ldots & 0 \\
0 & \alpha_{2,+} & \alpha_{3} & \alpha_{4,-} & 0 & \ldots & 0 \\
\vdots & & & & & \ddots & \\
0 & 0 & 0 & \ldots & \alpha_{N-2,+} & \alpha_{N-1} & \alpha_{N,-} \\
0 & 0 & 0 & \ldots & 0 & \alpha_{N-1,+} & \alpha_{N}
\end{array}\right]
$$

This channel model is generalised from the channel model introduced in [19], used to investigate the uplink Shannon capacity. The band diagonal structure of the channel matrix

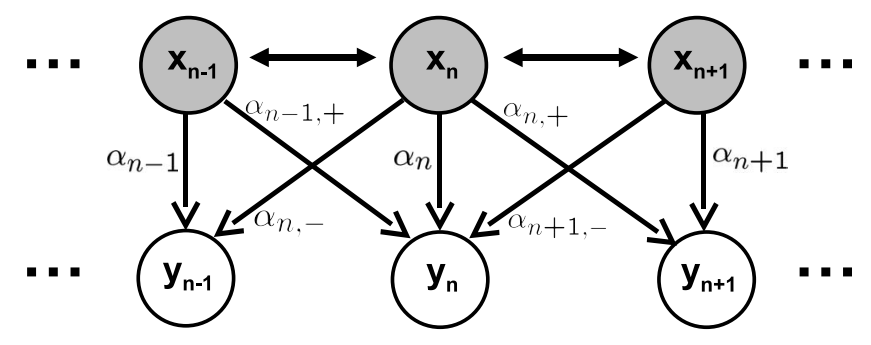

Fig. 1. Linear Array Model

provides a "local interaction" structure to the downlink beamforming problem, which we wish to exploit.

Denoting $\boldsymbol{T} \in \mathbb{R}^{N \times N}$ as the downlink beamformer matrix and $\boldsymbol{d}$ as the vector of data symbols intended for each MS, i.e. $\boldsymbol{d}=\left[\begin{array}{llll}d_{1} & d_{2} & \cdots & d_{N}\end{array}\right]^{T}$, the transmitted signal from each BS can be expressed as

$$
\boldsymbol{x}=\boldsymbol{T d} .
$$

The data symbols $\left\{d_{n}\right\}$ are assumed to be i.i.d. random variables with zero mean and unit variance, independent of $\left\{w_{n}\right\}$.

A particular type of transmit beamformer that has attracted much attention in the literature has the following form [2][13]:

$$
\boldsymbol{T}=\boldsymbol{H}^{T}\left(\boldsymbol{H} \boldsymbol{H}^{T}+\beta \boldsymbol{I}\right)^{-1} \boldsymbol{\Gamma}
$$

where $\beta$ is a positive scalar and $\Gamma \in \mathbb{R}^{N \times N}$ is a diagonal matrix, which can be seen as the scaling on the data symbols. We shall adopt the transmit beamformer structure (3) throughout this paper, leading to the transmitted symbol vector

$$
\begin{aligned}
\boldsymbol{x} & =\boldsymbol{H}^{T}\left(\boldsymbol{H} \boldsymbol{H}^{T}+\beta \boldsymbol{I}\right)^{-1} \boldsymbol{\Gamma} \boldsymbol{d} \\
& =\boldsymbol{H}^{T}\left(\boldsymbol{H} \boldsymbol{H}^{T}+\beta \boldsymbol{I}\right)^{-1} \tilde{\boldsymbol{d}}
\end{aligned}
$$

where we have simplified the expression by the substitution $\tilde{d}=\Gamma d$.

While we have assumed that the data symbols and path gains are real quantities in our signal model, it is worth noting that our results can easily be extended to the case of complex data symbols and channel gains.

\section{Downlink BeAmforming Problem as A Virtual LMMSE ESTIMATION PROBLEM}

We make the important observation that (5) resembles a well-known result in estimation theory: $\boldsymbol{x}$ can be seen as a LMMSE estimate of some vector $\boldsymbol{u}$ under the alternative model:

$$
\tilde{\boldsymbol{d}}=\boldsymbol{H} \boldsymbol{u}+\boldsymbol{z}
$$

where $\boldsymbol{u}=\left[\begin{array}{llll}u_{1} & u_{2} & \cdots & u_{N}\end{array}\right]^{T}$ is a vector of i.i.d. random variables with zero mean and unit variance and $\boldsymbol{z}=$ $\left[\begin{array}{llll}z_{1} & z_{2} & \cdots & z_{N}\end{array}\right]^{T}$ is a vector of i.i.d. random variables with zero mean and variance $\beta$, independent of $\boldsymbol{u}$. In (6), $\boldsymbol{u}$ can be interpreted as a data vector, $\boldsymbol{H}$ as a channel matrix, $\tilde{\boldsymbol{d}}$ 
as an observation data vector and $z$ as the additive noise, in a virtual model.

Note that $\boldsymbol{u}$ and $\boldsymbol{z}$ have no physical meaning in terms of our original model (1). However, a consequence of the above observation is that many signal processing techniques developed for LMMSE estimation problems (e.g. [15]) are readily applicable here. In particular, we are interested in demonstrating that the distributed estimation technique based on the Kalman smoothing framework developed in [15] can be applied, so that the downlink signal generation (5) can be implemented in a distributed manner using local message passing between BSs. Finally, we remark that (6) is different from the conventional virtual uplink model used to obtain the downlink beamformer based on the duality of multiple access channel and broadcast channel [11], [13], [20].

\section{Distributed Downlink BeAmforming}

\section{A. State Space Model and Kalman Smoothing}

In this section, we shall treat $\tilde{\boldsymbol{d}}$ as the observation data vector on $\boldsymbol{u}$ in accordance to the channel model (6) and $\boldsymbol{x}$ as the LMMSE estimate of $\boldsymbol{u}$ given $\tilde{\boldsymbol{d}}$. This virtual estimation problem can actually be formulated as a Kalman smoothing problem by exploiting the "local interaction" structure of the "channel". Since the Kalman smoothing problem formulation is identical to that presented for the uplink LMMSE estimation problem in [15], only an outline shall be presented here.

Treating the index $n$ as time, the structure of the channel matrix $\boldsymbol{H}$ allows us to formulate the following state space model:

$$
\left[\begin{array}{c}
u_{n} \\
u_{n+1} \\
u_{n+2}
\end{array}\right]=\left[\begin{array}{lll}
0 & 1 & 0 \\
0 & 0 & 1 \\
0 & 0 & 0
\end{array}\right]\left[\begin{array}{c}
u_{n-1} \\
u_{n} \\
u_{n+1}
\end{array}\right]+\left[\begin{array}{l}
0 \\
0 \\
1
\end{array}\right] u_{n+2}
$$

for $n=1, \ldots, N-1$. The observation model is given by

$$
\tilde{d}_{n}=\left[\begin{array}{lll}
\alpha_{n-1,+} & \alpha_{n} & \alpha_{n+1,-}
\end{array}\right]\left[\begin{array}{c}
u_{n-1} \\
u_{n} \\
u_{n+1}
\end{array}\right]+z_{n} .
$$

Given the above state space model, a forward Kalman filter can be applied [21] to produce the LMMSE estimate of the state vector $\left[\begin{array}{lll}u_{n-1} & u_{n} & u_{n+1}\end{array}\right]^{T}$, given $\left\{\tilde{d}_{1}, \cdots, \tilde{d}_{n}\right\}$ at a particular 'time' $n$. The forward Kalman filter is initialised by estimating $\left[\begin{array}{ll}u_{1} & u_{2}\end{array}\right]^{T}$ based on $\tilde{d}_{1}$.

One can also write down the state space model in the 'backward' direction:

$$
\left[\begin{array}{c}
u_{n-2} \\
u_{n-1} \\
u_{n}
\end{array}\right]=\left[\begin{array}{lll}
0 & 0 & 0 \\
1 & 0 & 0 \\
0 & 1 & 0
\end{array}\right]\left[\begin{array}{c}
u_{n-1} \\
u_{n} \\
u_{n+1}
\end{array}\right]+\left[\begin{array}{l}
1 \\
0 \\
0
\end{array}\right] u_{n-2}
$$

for $n=2, \ldots, N$. In this case, the corresponding backward Kalman filter produces at 'time' $n$, the LMMSE estimate of the state vector $\left[\begin{array}{lll}u_{n-1} & u_{n} & u_{n+1}\end{array}\right]^{T}$, given $\left\{\tilde{d}_{n}, \cdots, \tilde{d}_{N}\right\}$. The backward Kalman filter is initialised by estimating $\left[\begin{array}{ll}u_{N-1} & u_{N}\end{array}\right]^{T}$ based on $\tilde{d}_{N}$.

Using the fact that the Kalman smoother can be interpreted as as linear combination of two Kalman filters [22]; one which runs in the forward direction on the data set $\left\{\tilde{d}_{1}, \cdots, \tilde{d}_{n}\right\}$ and the other in the backward direction on $\left\{\tilde{d}_{n+1}, \cdots, \tilde{d}_{N}\right\}$, we now have an efficient mechanism to estimate the state vector $\left[\begin{array}{lll}u_{n-1} & u_{n} & u_{n+1}\end{array}\right]^{T}$ given all $\left\{\tilde{d}_{1}, \cdots, \tilde{d}_{N}\right\}$, which can be implemented via message passing as outlined in the next section.

\section{B. Forward-backward Beamforming Algorithm}

A natural distributed beamforming algorithm based on the Kalman smoothing framework described in the previous section is the forward-backward algorithm. We provide a high level description of the forward-backward beamforming algorithm below; interested readers are referred to [15] from which the precise equations for the updates can be inferred.

1) At both ends of the linear array, the initial estimates of $\left\{u_{1}, u_{2}\right\}$ and $\left\{u_{N-1}, u_{N}\right\}$ are produced by $B S 1$ (given the data $\tilde{d}_{1}$ ) and $B S N$ (given the data $\tilde{d}_{N}$ ) respectively. This is the initialisation step of the forward and backward Kalman filters;

2) The outputs of BS 1 and BS $N$ are passed as messages to their direct neighbours, namely $B S 2$ and $B S N-$ 1. Then, BS 2 and BS N-1 incorporate the messages received with the information from their own data $\tilde{d}_{2}$ and $\tilde{d}_{N-1}$ to produce new messages for BS 3 and BS $N-2$ respectively;

3) The process of information incorporation and message passing continues in both the forward and the backward directions until all BSs have received messages from both their left and right neighbours;

4) For each $n$, the messages at BS $n$ are then linearly combined to produce $x_{n}$;

An important remark is that the distributed algorithm outlined above is sensitive to the size of the network. The time required to propagate information to cover the entire network increases linearly with the size of the array.

\section{Limited Extent Distributed Beamforming Algorithm}

Equation (5) suggests that to obtain $x_{n}$, we need all $\tilde{d}_{m} \mathrm{~s}$, i.e. all scaled data symbols to be transmitted in the network. However, due to the highly localised intercell interference, as exhibited in the tridiagonal structure of the channel matrix, one expects $\tilde{d}_{m}$ to become less important for the generation of $x_{n}$ as the distance between BS $m$ and BS $n$ increases. This is reflected in the small weights that are applied to the data symbols from BSs far away. For illustrative purposes, we calculate the weights according to (3) for a fixed $\boldsymbol{H}$ and for $\boldsymbol{\Gamma}$ and $\beta$ obtained from [2]-[4], i.e. $\boldsymbol{\Gamma}=r \boldsymbol{I}$, where $r$ is a normalising factor such that the average total transmit power is some value $P_{t}$, and $\beta=N \sigma^{2} / P_{t}$. The result, shown in Fig. 2, implies that there is little loss in performance if data symbols from BSs sufficiently far away are ignored. This results in a limited extent distributed algorithm which is outlined below; again, the details can be inferred from [15]. By symmetry, without loss of generality we can describe the algorithm from the point of view of a particular BS at cell $n$. 


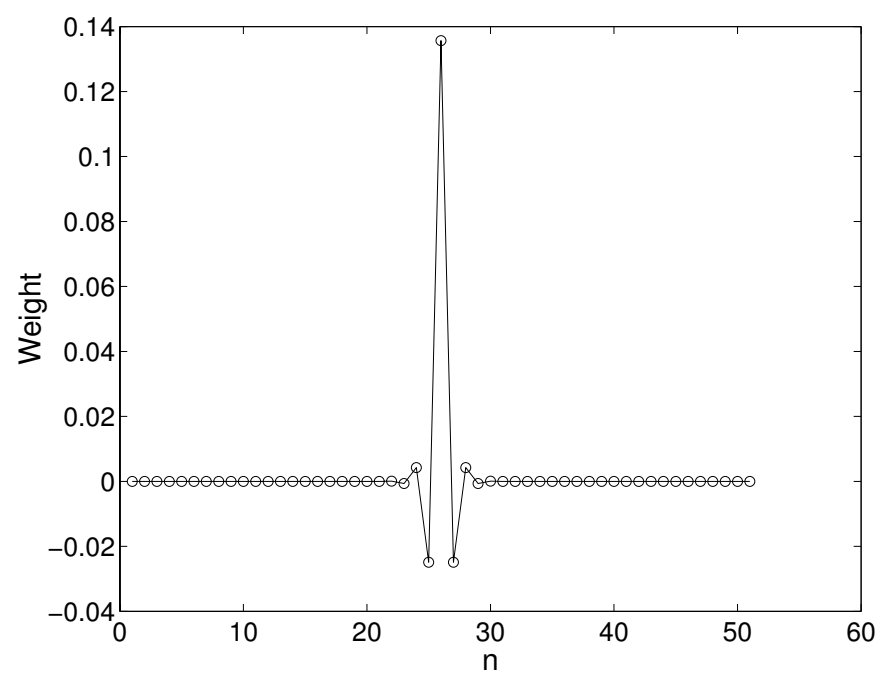

Fig. 2. Weights on each data symbol for central BS in an array of size 51 $\left(\alpha_{n}=1, \alpha_{n,+}=\alpha_{n,-}=0.2, \sigma^{2}=0.001\right.$ and $\left.P_{t}=1\right)$.

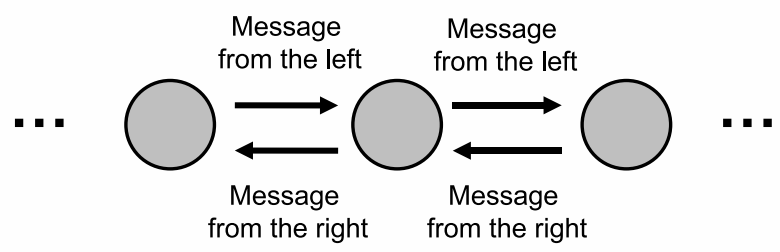

Fig. 3. Message passing between transmitter nodes

1) $\left\{u_{n-1}, u_{n}, u_{n+1}\right\}$ are estimated based on the data $\tilde{d}_{n}$;

2) The initial estimates of $\left\{u_{n-1}, u_{n}\right\}$ are sent to the $B S$ $n-1$, while the initial estimates of $\left\{u_{n}, u_{n+1}\right\}$ are sent to the $B S n+1$. At the same time, messages corresponding to the initial estimates by the neighbours, $B S n-1$ and $B S n+1$, are received;

3) The message received from the left neighbour, $B S n-1$, is incorporated with $B S$ n's own information, $\tilde{d}_{n}$, and the result is then passed to the right neighbour, $B S n+1$. Similarly, the message received from the right neighbour is incorporated with $\tilde{d}_{n}$ and the result is passed to the left (Fig. 3);

4) After $j$ passes, the estimate of $u_{n}$ based on $\left\{\tilde{d}_{n-j}, \cdots, \tilde{d}_{n+j}\right\}$ can be obtained. This provides $x_{n}^{(j)}$, the approximation (after $j$ passes) to $x_{n}$.

Observe that the algorithm can be terminated after an arbitrary number of passes, and the $x_{n}^{(j)}$ obtained incorporates only the data symbols from the limited extent set corresponding to the number of passes that have taken place (Fig. 4). Ultimately, if the number of passes is large enough for the information from the BSs at the edges of the array to arrive, then the resultant $x_{n}$ is optimal. Obviously, the saving on delay depends on the number of passes needed to achieve a certain

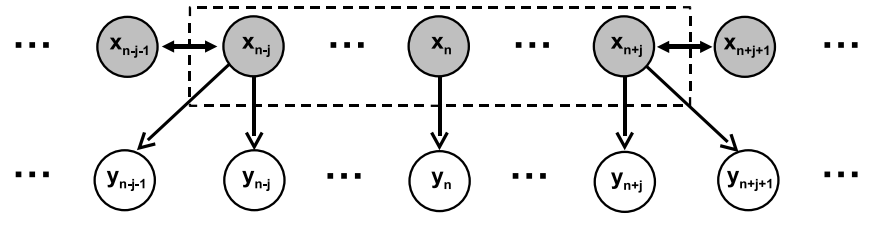

Fig. 4. Limited extent set of BSs associated with generation of $x_{n}$ after $j$ message-passing steps. Only $\left\{d_{n-j}, \cdots d_{n+j}\right\}$ are involved in producing $x_{n}$

performance; the lower the number passes required, the lower the delay.

\section{Performance Comparison in Rayleigh Fading Environment}

In this section, we present some simulation results to compare the performance of the forward-backward and the limited extent algorithm. The simulation set up is as follows:

- the number of BSs in the linear array is $N=51$;

- $\left\{\alpha_{n,-}, \alpha_{n}, \alpha_{n,+}\right\}$ are independent Rayleigh random variables with $E\left[\alpha_{n}^{2}\right]=1$ and $E\left[\alpha_{n,+}^{2}\right]=E\left[\alpha_{n,-}^{2}\right]=\alpha^{2}$;

- the noise variance at each MS is $\sigma^{2}$ and the total transmit power is $P_{t}=N$; and

- the transmit beamformer to be implemented (or approximated) is [2]-[4] $\boldsymbol{T}=r \boldsymbol{H}^{T}\left(\boldsymbol{H} \boldsymbol{H}^{T}+\beta \boldsymbol{I}\right)^{-1}$ where $\beta=N \sigma^{2} / P_{t}=\sigma^{2}$ and $r$ is a normalising constant.

We study the SINR achieved by the MS in the middle of the linear array. It is of interest to plot the SINR achieved by the limited extent algorithm with respect to the number of message passing steps performed. For fair comparison, we always scale the transmitted vector so that the total transmit power is the same for all cases.

Fig. 5 and Fig. 6 illustrates the SINR achieved by the middle MS after a certain number of message passing steps, averaged over 10000 channel realisations. The dashed line is the SINR produced by the complete forward-backward algorithm. Fig. 5 corresponds to a cellular network with moderate intercell interference, whereas Fig. 6 corresponds to the case of high intercell interference. Not surprisingly, the convergence to the optimal SINR is faster for smaller intercell interference, however even in the case of quite severe intercell interference, a SINR within $10 \%$ of the final value is reached after five message passing iterations.

\section{CONCLUSION}

Two distributed beamforming algorithms based on the Kalman smoothing framework that efficiently calculate the transmitted signal of each BS in a one-dimensional cellular network are presented. The first algorithm is a forwardbackward algorithm that produces exactly the same transmitted signal that would be produced by a centralised beamformer. However, the algorithm suffers from a delay that grows linearly with the network size. The limited extent distributed beamforming is developed to overcome the delay problem by recognising the fact that only data symbols in the local neighbourhood are important for generating the signal for transmission. 


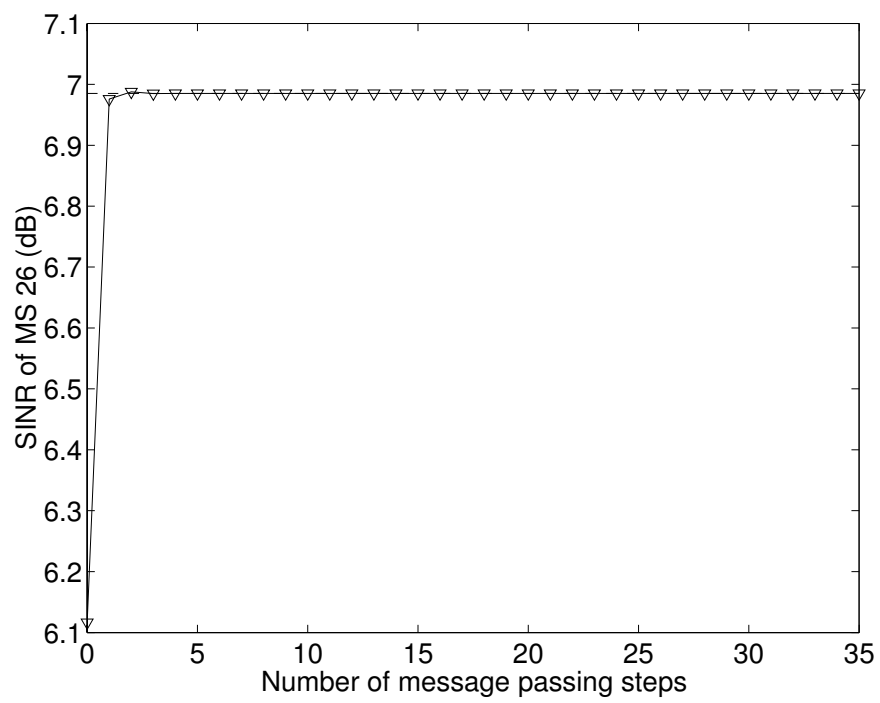

Fig. 5. SINR of central MS versus the number of message passing steps in an array of size $51\left(\alpha=0.2, \sigma^{2}=0.1, P_{t}=N\right)$.

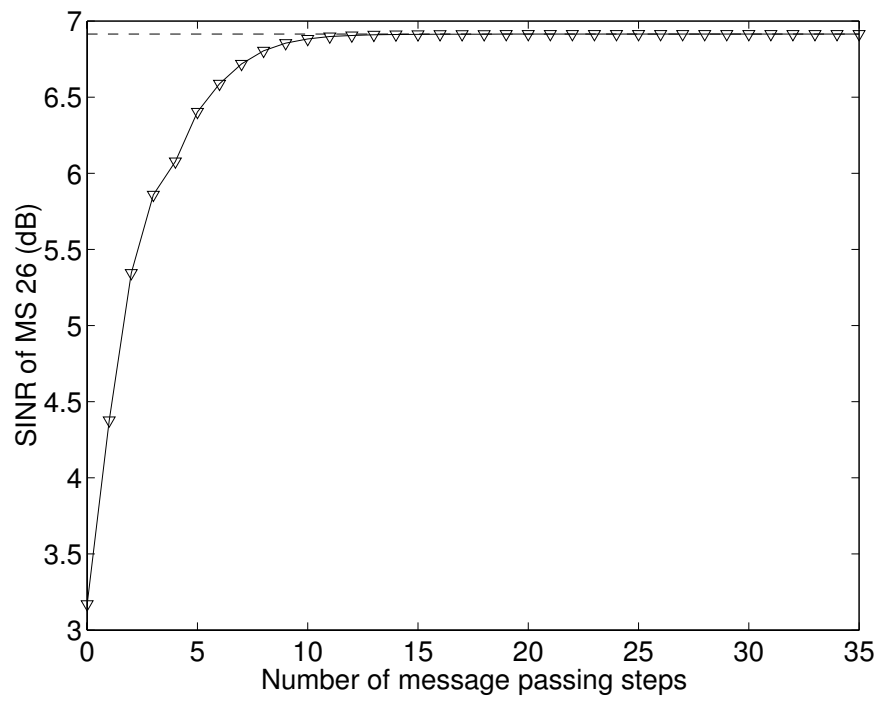

Fig. 6. SINR of central MS versus the number of message passing steps in an array of size $51\left(\alpha=0.9, \sigma^{2}=0.1, P_{t}=N\right)$.

\section{ACKNOWLEDGMENT}

B. Ng, J. Evans and S. Hanly are with the ARC Special Research Centre for Ultra-Broadband Information Networks (CUBIN), an affiliated program of National ICT Australia. This work was supported by the Australian Research Council.

\section{REFERENCES}

[1] S. Shamai, O. Somekh, and B. M. Zaidel, "Multi-cell communications: An information theoretic perspective," in Joint Workshop on Communications and Coding, Donnini (Florence), Italy, Oct. 2004.

[2] J. A. Nossek, M. Joham, and W. Utschick, "Transmit processing in MIMO wireless systems," in Proc. 6th CAS Symposium on Emerging Technologies, vol. 1, May 2004, pp. I-18 - I-23.

[3] M. Joham, K. Kusume, M. H. Gzara, W. Utschick, and J. A. Nossek, "Transmit Wiener filter for the downlink of TDDDS-CDMA systems," in Proc. IEEE 7th Int. Symp. on Spread-Spectrum Tech. and Appl., Prague, Czech Republic, vol. 1, Sept. 2002, pp. 9 - 13.
[4] L.-U. Choi and R. D. Murch, "Transmit-preprocessing techniques with simplified receivers for the downlink of MISO TDD-CDMA systems," IEEE Trans. on Vehicular Technology, vol. 53, no. 2, pp. 285 - 295 , Mar. 2004.

[5] A. N. Barreto and G. Fettweis, "Capacity increase in the downlink of spread spectrum systems through joint signal precoding," in Proc. International Conference on Communications, Helsinki, Finland, vol. 4 Jun. 2001, pp. $1142-1146$.

[6] F. Kowalewski and P. Mangold, "Joint predistortion and transmit diversity," in Proc. IEEE Global Telecommunications Conference, vol. 1, Nov. 2000, pp. $245-249$.

[7] B. R. Vojčić and W. M. Jang, "Transmitter precoding in synchronous multiuser communications," IEEE Trans. Commun., vol. 46, no. 10, pp. 1346 - 1355, Oct. 1998.

[8] A. Wiesel, Y. C. Eldar, and S. Shamai, "Linear precoding via conic optimization for fixed MIMO receivers," to appear in IEEE Trans. Signal Processing.

[9] M. Schubert and H. Boche, "Solution of the multiuser downlink beamforming problem with individual SINR constraints," IEEE Trans. on Vehicular Technology, vol. 53, no. 1, pp. 18 - 28, Jan. 2004.

[10] H. Boche and M. Schubert, "A general duality theory for uplink and downlink beamforming," in Proc. IEEE Vehicular Technology Conference (VTC-2002 Fall), Sept. 2002, pp. 87 - 91.

[11] F. Rashid-Farrokhi, K. J. R. Liu, and L. Tassiulas, "Transmit beamforming and power control for cellular wireless systems," IEEE Journal on Selected Areas in Communications, vol. 16, no. 8, pp. 1437 - 1450, Oct. 1998.

[12] F. Rashid-Farrokhi, L. Tassiulas, and K. R. Liu, "Joint optimal power control and beamforming in wireless networks using antenna arrays," IEEE Tran. Commun., vol. 46, no. 10, pp. 1313 - 1324, Oct. 1998.

[13] E. Visotsky and U. Madhow, "Optimum beamforming using transmit antenna arrays," in Proc. IEEE 49th Vehicular Technology Conference, vol. 1, May 1999, pp. $851-856$.

[14] C. B. Peel, B. M. Hochwald, and A. L. Swindlehurst, "A vectorperturbation technique for near-capacity multi-antenna multi-user communication-part I: Channel inversion and regularization," IEEE Trans. on Communications, to appear.

[15] B. L. Ng, J. S. Evans, and S. V. Hanly, "Distributed linear multiuser detection in cellular networks based on Kalman smoothing," in Proc. IEEE Global Telecommunications Conference, Dallas, USA, vol. 1, Dec. 2004, pp. $134-138$.

[16] S. Shamai and B. M. Zaidel, "Enhancing the cellular downlink capacity via co-processing at the transmitting end," in Proc. IEEE VTC 2001 Spring, Rhodes, Greece, vol. 3, May 2001, pp. 1745 - 1749.

[17] S. A. Jafar and A. J. Goldsmith, "Transmitter optimization for multiple antenna cellular systems," in Proc. IEEE International Symposium on Information Theory, Lausanne, Switzerland, June 2002, p. 50.

[18] A. Ekbal and J. M. Cioffi, "Distributed transmit beamforming in cellular networks - a convex optimization perspective," Proc. IEEE International Conference on Communications, May 2005.

[19] A. D. Wyner, "Shannon-theoretic approach to a Gaussian cellular multiple-access channel," IEEE Tran. Inform. Theory, vol. 40, pp. 1713 1727, Nov. 1994.

[20] D. N. C. Tse and P. Viswanath, "Downlink-uplink duality and effective bandwidths," in Proc. IEEE International Symposium on Information Theory, Lausanne, Switzerland, June 2002, p. 52.

[21] S. M. Kay, Fundamentals of Statistical Signal Processing - Estimation Theory. Prentice Hall, 1993.

[22] D. C. Fraser and J. E. Potter, "The optimum linear smoother as combination of two optimum linear filters," IEEE Trans. Automatic Control, pp. 387-390, Aug. 1969. 